\title{
Cultura tributaria en el Ecuador y sostenibilidad fiscal
}

\author{
Fecha de recepción: 31/10/2019 • Fecha de aceptación: 10/12/2019 • Fecha de publicación:10/02/2020
}

\author{
Andrade Donoso María Soledad \\ Textiles Texsa \\ sandrade@textilestexsa.com \\ https://orcid.org/0000-0003-0706-0340 \\ Cevallos Caza Katya Geovanna \\ katylu2003@hotmail.com \\ https://orcid.org/0000-0002-4686-8265
}

\section{Resumen}

Es de conocimiento público la importancia que ha adquirido en los últimos años para la sostenibilidad fiscal del Ecuador el rubro de los impuestos. En este artículo se expondrá el peso de cada uno de los impuestos en la recaudación anual (2017 y 2018), con la finalidad de indagar sobre la cultura tributaria en el país. Se fundamenta teóricamente, mediante conceptos como: cultura tributaria obligaciones, impuestos, estructura de los contribuyentes. Para ello se llevó a cabo una investigación documental, en libros y textos académicos, los datos expresados son cuantitativos, a través de cuadros estadísticos de las páginas del SRI y del INEC. El resultado que se obtuvo de la investigación es que el Ecuador obtiene la mayoría de sus ingresos fiscales del cobro de impuestos, duplicando los ingresos por exportaciones petroleras, por lo cual son el fundamento para la sostenibilidad fiscal de allí que se debe fomentar la cultura tributaria para contribuir al desarrollo del país, puesto que existe aún una proporción significativa de recaudación por intereses de mora y por multas tributarias, que incluso superan la recaudación por RISE, que paradójicamente conforman más de la mitad del total de empresas en el país. 


\begin{abstract}
It is of public knowledge the importance that has acquired in the last years for the fiscal sustainability of Ecuador the heading of the taxes. This article will expose the weight of each of the taxes in the annual collection (2017 and 2018), in order to inquire about the tax culture in the country. It is theoretically based, through concepts such as: tax culture obligations, taxes, structure of taxpayers. For this, a documentary investigation was carried out, in books and academic texts, the data expressed are quantitative, through statistical tables of the pages of the SRI and INEC. The result that was obtained from the investigation is that Ecuador obtains the majority of its fiscal income from the collection of taxes, doubling the revenues from oil exports, which is why they are the basis for the fiscal sustainability of that tax culture should be encouraged. to contribute to the development of the country, since there is still a significant proportion of collection for interest on arrears and tax fines, which even exceed the collection by RISE, which paradoxically make up more than half of the total number of companies in the country.
\end{abstract}

KEYWORDS: taxes, tax culture, collection, companies 


\section{Introducción}

Han pasado varios siglos desde que esta frase fue pronunciada, "En este mundo no hay nada cierto, excepto la muerte y los impuestos", Benjamín Franklin (1706-1790),pero sigue vigente y mientras el funcionamiento económico de los estados dependa de los tributos, estos nunca dejarán de existir y no lo harán ya que los gobiernos necesitan de estos fondos para financiar las necesidades básicas del pueblo.

En el Ecuador la cultura tributaria está encontrando cimiento en los últimos años apenas, por experiencias pasadas los ecuatorianos piensan que los fondos públicos siempre se van a despilfarrar y no se los va a invertir en el pueblo, por este motivo y por la idiosincrasia popular los ecuatorianos preferían buscar todas las maneras posibles para evadir impuestos, a esto se le suma un sistema de control débil y permisivo.

Todo esto se está intentado transformar en un contexto de reformas de fondo y forma en el Código Tributario y de un profundo cambio tecnológico en todas las plataformas del Gobierno que intervienen en el procesamiento de las obligaciones tributarias (SRI, SENAE).

Pero lo mas importante es que la razón prive sobre la coerción estatal y que los ciudadanos tenga cultura tributaria, puesto que la recaudación tributaria es el principal cimiento de ingresos fiscales para la sostenibilidad fiscal.

\section{Obligación tributaria}

El Art.15 del Código Tributario expresa que: "obligación tributaria es el vínculo jurídico personal, existente entre el Estado o las entidades acreedoras de tributos y los contribuyentes o responsables de aquellos, en virtud del cual debe satisfacerse una prestación en dinero, especies o servicios apreciables en dinero, al verificarse el hecho generador previsto por la Ley".

Los elementos indispensables para que exista o concurra una obligación tributaria son los siguientes:

- Hecho Generador. - Dentro del Art. 16 del Código Tributario expresa que se entiende por hecho generador al presupuesto establecido por la ley para configurar cada tributo.Por lo expresado al hecho generador se entiende al presupuesto del hecho, como la hipótesis legal que es el fundamento o soporte constitutivo de la obligación cuya obtención o ejecución está unida a su nacimiento, por lo tanto, instituye el nacimiento de una actividad económica.

- Sujeto Activo. - El Art. 23 del Código Tributario pronuncia que sujeto activo es el ente público acreedor del tributo. Es el ente acreedor o consignatario del tributo, puede ser el Estado (administrado por el SRI), Municipios, Consejos Provinciales, o cualquier ente merecedor del tributo.

- Sujeto Pasivo. - El Art. 24 del Código Tributario profiere: es sujeto pasivo la persona natural o jurídica que, según la Ley, está obligada al cumplimiento de la prestación tributaria, sea como 
contribuyente o como responsable. Es la persona natural o jurídica que esta compelida o constreñida al cumplimiento de las prestaciones tributarias, sean como contribuyente o como responsable de la obligación tributaria.

En el Código Tributario, Registro Oficial 405, del 29 de diciembre del 2014. Libro Segundo De los Procedimientos Tributarios, Título I Del Procedimiento Administrativo Tributario, Capítulo III Deberes Formales del Contribuyente o Responsable, Código Tributario, Reformado (2016)

"Art. 96.- Deberes formales. - Son deberes formales de los contribuyentes o responsables:

1. Cuando lo exijan las leyes, ordenanzas, reglamentos o las disposiciones de la respectiva autoridad de la administración tributaria:

a) Inscribirse en los registros pertinentes, proporcionando los datos necesarios relativos a su actividad; y, comunicar oportunamente los cambios que se operen;

b) Solicitar los permisos previos que fueren del caso;

c) Llevar los libros y registros contables relacionados con la correspondiente actividad económica, en idioma castellano; anotar, en moneda de curso legal, sus operaciones o transacciones y conservar tales libros y registros, mientras la obligación tributaria no esté prescrita;

d) Presentar las declaraciones que correspondan; y,

e) Cumplir con los deberes específicos que la respectiva ley tributaria establezca.

\section{Cultura tributaria}

Es una conducta manifestada en el cumplimiento permanente de los deberes tributarios con base en la razón, la confianza, la afirmación de valores de ética personal, respeto a la ley, responsabilidad ciudadana y solidaridad social de los contribuyentes. No sólo es una obligación fiscal, sino un deber ciudadano para lograr el desarrollo del país, mediante una actitud responsable motivada por creencia y valores, aceptando el deber de contribuir a que el Estado cumpla sus fines.

Los recursos que el Estado necesita para cumplir con su obligación constitucional de velar por el bien común y proporcionar a la población los servicios básicos provienen esencialmente de los tributos pagados por los contribuyentes. Para ello El Estado puede apelar a la coerción mediante la presión ejercida por las leyes y en su cumplimiento obligatorio, o a la razón dada por una Cultura Tributaria con bases sólidas.

De allí la importancia del manejo y destino de los fondos públicos, lo cual genera una percepción positiva o negativa sobre el uso de los fondos. A efecto de fortalecer la Cultura Tributaria, se requiere que la población obtenga información oportuna y formación adecuada, en función de 
la concienciación, que redundará en una recaudación firme y sostenible. Consecuentemente se puede decir que los ejes centrales para la promoción de la Cultura Tributaria son la Información, la Formación y la Concienciación, los cuales se articulan en torno a la razón como móvil deseable fundamental de la acción de tributar. (Cultura tributaria, 2012)

En cuanto a la coerción que efectúa la autoridad tributaria para el cumplimiento de las obligaciones tributarias se señala que:

Se requiere optimizar los procesos de control del cumplimiento de las obligaciones impositivas, tanto en lo que se refiere al control extensivo, como al realizado mediante procesos de inteligencia fiscal (control intensivo); en este último caso es necesario reducir al máximo la discrecionalidad en la selección de los casos a fiscalizar, a fin de obtener los mejores resultados. El uso de las herramientas que la tecnología proporciona hoy en día, especialmente las informáticas, es de vital importancia para facilitar el cumplimiento de las obligaciones tributarias y optimizar el control de las mismas. Conferencia técnica Administración Tributaria Viable, Estado Viable, 2005)

\section{Sostenibilidad fiscal}

La sostenibilidad fiscal significa, en esencia, que el gasto público no debe crecer por encima de los ingresos. De esta forma se evita que el Estado, para financiar sus egresos, tenga que endeudarse cada vez más, y que la deuda pública crezca de forma desmedida, excediendo la capacidad de pago del país.

\section{Los impuestos}

Según el SRI, (s/f) la recaudación por los siguientes rubros se conceptualiza de la siguiente forma:

- Impuesto a la renta. Se aplica sobre aquellas rentas que obtengan las personas naturales, las sucesiones indivisas y las sociedades sean nacionales o extranjeras. El ejercicio impositivo comprende del 1 de enero al 31 de diciembre.

- El Impuesto al valor agregado IVA. Es una carga fiscal sobre el consumo, es decir, financiado por el consumidor como impuesto regresivo, aplicado en muchos países y generalizado en la Unión Europea. Wikipedia

- Interés por mora tributario. Es la prestación accesoria que se exige a los obligados tributarios y a los sujetos infractores como consecuencia de la realización de un pago fuera de plazo.

- Las multas tributarias fiscales. Son las sanciones pecuniarias por incumplimientos tributarios, que puede ser por no presentar declaraciones de impuestos, no exhibir la documentación solicitada por el SRI en una auditoría, presentar las declaraciones de manera parcial, con errores o tardíamente, La presentación por fuera del período legal de los anexos de gastos personales, la Rebeldía, es decir no acude a las comparecencias solicitadas por la autoridad tributaria, etc. 


\section{Empresas según su forma institucional}

El SRI (s/f) tiene una clasificación de las empresas según su forma institucional, la cual se describe a continuación

- El Régimen Impositivo Simplificado (RISE): es un régimen de inscripción voluntaria, que reemplaza el pago del IVA y del Impuesto a la Renta a través de cuotas mensuales y tiene por objeto mejorar la cultura tributaria en el país. Los requisitos son

- Ser persona natural.

- No tener ingresos mayores a USD 60 mil en el año, o si se encuentra bajo relación de dependencia el ingreso por este concepto no supere la fracción básica del Impuesto a la Renta gravado con tarifa cero por ciento $(0 \%)$ para cada año.

- No dedicarse a alguna de las actividades restringidas.

- No haber sido agente de retención durante los últimos 3 años.

- Personas naturales obligadas a llevar contabilidad: Son todas las personas nacionales y extranjeras que realizan actividades económicas y que cumplen con las siguientes condiciones:

- Que operen con un capital propio que al inicio de sus actividades económicas o al 10. de enero de cada ejercicio impositivo

- Hayan superado 9 fracciones básicas desgravadas del impuesto a la renta o cuyos ingresos brutos anuales de esas actividades,

- Del ejercicio fiscal inmediato anterior, hayan sido superiores a 15 fracciones básicas desgravadas o cuyos costos y gastos anuales,

- Imputables a la actividad empresarial, del ejercicio fiscal inmediato anterior hayan sido superiores a 12 fracciones básicas desgravadas.

En estos casos, están obligadas a llevar contabilidad, bajo la responsabilidad y con la firma de un contador legalmente autorizado e inscrito en el Registro Único de Contribuyentes (RUC), por el sistema de partida doble, en idioma castellano y en dólares de los Estados Unidos. (Maldonado P. y De la Torre C., 2016)

Personas naturales no obligadas a llevar contabilidad: Son las que no cumplan con lo anterior, así como los profesionales, comisionistas, artesanos, y demás trabajadores autónomos (sin título profesional y no empresarios), no están obligados a llevar contabilidad, sin embargo deberán llevar un registro de sus ingresos y egresos. (Maldonado P. y De la Torre C., 2016)

- Organizaciones con fines de lucro: Llamadas empresas, tienen como uno de sus principales 
fines (si no es el único) generar una determinada ganancia o utilidad para su(s) propietario(s) y/o accionistas. (Thompson, 2017)

- Organizaciones sin fines de lucro: Se caracterizan por tener como fin cumplir un determinado rol o función en la sociedad sin pretender una ganancia o utilidad por ello. . Por el contrario, se trata de un objetivo social, artístico o que busca el bienestar comunitario. El ejército, la Iglesia, los servicios públicos, las entidades filantrópicas, las organizaciones no gubernamentales (ONG), etc. [1] son ejemplos de este tipo de organizaciones. (Thompson, 2017)

- Instituciones Públicas: Son unidades económicas dedicadas a la prestación de servicios por parte de los diferentes niveles de gobierno (federal, estatal o municipal). Incluye poder judicial, poder legislativo, instituciones autónomas de educación superior, así como otras de interés público y de carácter no educativo, y cualquier otra institución que opere con fondos y/o patrimonio públicos. Se clasifican en instituciones administradas por el gobierno y no administradas por el gobierno. (Romero, s/f))

\section{Metodología}

Para realizar este artículo el estudio se enmarco en un tipos documental, de carácter descriptivo y con enfoque cuantitativo (Bernal, 2010). Se utilizó la una investigación documental, extrayendo información de libros, artículos, revistas científicas, legislación, páginas web, etc.

Mediante el método inductivo, después de analizar hechos particulares se llega a una conclusión general sobre la cultura tributaria actual del país.

La investigación fue desarrollada mediante un enfoque cuantitativo, puesto que se examina datos referidos a impuestos, empresas, cuyas fuentes de información son organismos oficiales como el Servicio de Rentas Internas (SRI) y el Instituto Nacional de Estadística y Censos del Ecuador (INEC)

El estudio es de alcance descriptivo, puesto que se enfoca en describir la conformación de la recaudación en los años 2017 y 2018, para indagar sobre la cultura tributaria en el país, dada la importancia de los tributos en la sostenibilidad fiscal

\section{Resultados}

Para realizar el análisis se presenta la conformación de la recaudación de los años 2017 y 2018, de acuerdo a los tipos de impuesto recaudado y el monto por cada uno de estos rubros. En el cuadro, publicado por el SRI se puede apreciar estos recaudos 


\section{Cuadro 1}

Recaudación Nacional

\section{Miles de dólares}

2017-2018

\section{RECAUDACIÓN NACIONAL \\ ENERO - DICIEMBRE 2017 - 2018 -miles de dólares-}

\begin{tabular}{|c|c|c|c|}
\hline \multicolumn{2}{|r|}{ 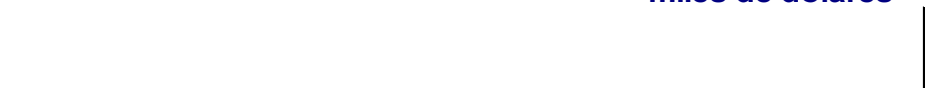 } & $\begin{array}{l}\text { Recaudación Enero - } \\
\text { Diciembre } 2017\end{array}$ & \begin{tabular}{|c|} 
Recaudación Enero - \\
Diciembre $2018^{\left({ }^{A}\right)}$
\end{tabular} \\
\hline \multicolumn{2}{|r|}{$\begin{array}{l}\text { TOTAL RECAUDACIÓN SIN CONTRIBUCIONES } \\
\text { SOLIDARIAS }{ }^{(2)}\end{array}$} & 13.224.892 & 15.130 .095 \\
\hline \multirow{22}{*}{$\begin{array}{l}\text { 응 } \\
\frac{0}{8} \\
\frac{0}{1} \\
\frac{10}{5} \\
\frac{1}{0}\end{array}$} & Impuesto a la Renta Recaudado & 4.177 .023 & 5.319 .721 \\
\hline & Retenciones Mensuales ${ }^{(3)}$ & 2.641 .586 & 2.938 .754 \\
\hline & Anticipos al IR & 342.893 & 352.774 \\
\hline & Declaraciones de Impuesto a la Renta ${ }^{(4)}$ & 1.192 .545 & 2.028 .192 \\
\hline & Personas Naturales & 175.500 & 192.885 \\
\hline & Personas Jurídicas & 991.401 & 1.808 .326 \\
\hline & Herencias, Legados y Donaciones & 25.644 & 26.982 \\
\hline & IVA de Operaciones Internas & 4.671.557 & 4.789.094 \\
\hline & ICE de Operaciones Internas & 740.547 & 714.379 \\
\hline & Impuesto Ambiental Contaminación Vehicular & 110.952 & 119.487 \\
\hline & Impuesto Redimible Botellas Plásticas no Retornable & 31.172 & 34.785 \\
\hline & Impuesto a los Vehículos Motorizados & 191.480 & 214.621 \\
\hline & Impuesto a la Salida de Divisas & 1.097 .642 & 1.206 .090 \\
\hline & Impuesto Activos en el Exterior & 34.876 & 29.594 \\
\hline & RISE & 22.105 & 22.836 \\
\hline & Regalías, patentes y utilidades de conservación miner & 52.965 & 55.976 \\
\hline & Tierras Rurales & 7.313 & 2.345 \\
\hline & Contribución para la atención integral del cancer & 96.677 & 104.270 \\
\hline & Intereses por Mora Tributaria & 78.417 & 186.617 \\
\hline & Multas Tributarias Fiscales & 49.583 & 43.492 \\
\hline & Otros Ingresos & 8.180 & 68.639 \\
\hline & SUBTOTAL & 11.370 .490 & 12.911 .946 \\
\hline \multirow{3}{*}{ 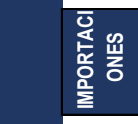 } & IVA de Importaciones & 1.645 .546 & 1.947 .054 \\
\hline & ICE de Importaciones & 208.855 & 271.095 \\
\hline & SUBTOTAL & 1.854.401 & 2.218 .150 \\
\hline \multirow{6}{*}{ 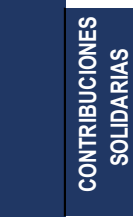 } & Contribución solidaria sobre el patrimonio & 15.969 & 6.002 \\
\hline & Contribución solidaria sobre las utilidades & 18.950 & 4.269 \\
\hline & Contribución solidaria sobre bienes de personas jurídi & 2.614 & 1.093 \\
\hline & Contribución solidaria sobre las remuneraciones & 4.852 & \\
\hline & Contribución 2\% IVA & 412.316 & 3.525 \\
\hline & SUBTOTAL & 454.701 & 14.974 \\
\hline \multicolumn{2}{|r|}{$\begin{array}{l}\text { TOTAL RECAUDACIÓN CON CONTRIBUCIONES } \\
\text { SOLIDARIAS }^{(2)}\end{array}$} & 13.679.593 & 15.145 .069 \\
\hline \multicolumn{2}{|r|}{ DIRECTOS } & 6.381 .916 & 7.388.661 \\
\hline \multicolumn{2}{|r|}{ INDIRECTOS } & 7.297.677 & 7.756 .408 \\
\hline \multirow{6}{*}{$\frac{\text { 出 }}{\frac{1}{6}}$} & TOTAL RECAUDACIÓN (2) & 13.679 .593 & 15.145 .069 \\
\hline & (-) Notas de Crédito & 716.262 & 713.545 \\
\hline & (-) Compensaciones & 37.419 & 112.567 \\
\hline & TOTAL EFECTIVO ${ }^{(5)}$ & 12.925 .911 & 14.318.957 \\
\hline & (-) Devoluciones ${ }^{(6)}$ & 197.120 & 241.329 \\
\hline & TOTAL NETO ${ }^{(7)}$ & 12.728 .791 & 14.077.628 \\
\hline
\end{tabular}

Fuente: Estadísticas Generales de Recaudación SRI (s/f) 
Al realizar un análisis vertical tanto del año 2017, como del 2018, se puede notar que la mayor proporción de la recaudación corresponde al Impuesto a la Renta y al IVA de operaciones internas que equivalen en ambos anos al $67 \%$ de total anual recaudado, mientras que el RISE corresponde e una ínfima proporción que no llega al 0,17 en 2017 y 0,15 en 2018 \%, lo que representa un porcentaje que no llega ni al 0,20 \% en ambos años.

También es importante resaltar que los rubros de Interese por mora tributaria y multas tributaria fiscales en el año 2017 alcanzan en conjunto el 0,97\% del total de la recaudación, asimismo en el año 2018 estos dos rubros equivalen al 1,52\% del total recaudado. Lo cual es contraproducente, puesto que se está recaudando más por incumplimiento tributario que por RISE.

Además, al realizar un análisis horizontal para ver la variabilidad entre al año 2017 y el 2018, se puede notar la mayor variación es la del Impuesto sobre la Renta cuyo incremento de un año a otro es del $27 \%$, mientas que el IVA y el RISE solo tuvieron un incremento del $3 \%$. Asimismo es de hacer notar que el monto por Intereses por mora tributaria, se incrementó en $138 \%$ de un año a otro, mientras que el de multas tributarias tuvo un decremento del $12 \%$

Estos resultados ocurre en un contexto en el cual, el Producto Interno Bruto (PIB) en el año 2017 en términos corrientes alcanzó USD 103,057 millones y en el 2018 fue de USD 107,562 millones, (Banco Central del Ecuador). Este dinamismo se explica principalmente por el aumento del Gasto por Consumo en los hogares, el Gasto de Consumo Final del Gobierno General y las Exportaciones.

Es importante, destacar que el monto de ingresos por exportaciones petroleras está por debajo de la recaudación tributaria, tal como se muestra en el cuadro 2, esto devela cuan significativo son los impuestos para el desarrollo del país, 
Cuadro 2

Exportaciones nacionales de crudo 2017-2018

EXPORTACIONES NACIONALES DE CRUDO

\begin{tabular}{|c|c|c|c|c|c|c|}
\hline Mes/Año & & 2017 & & & 2018 & \\
\hline & $\begin{array}{r}\text { VOLUMEN } \\
\text { Millones } \\
\text { Bls. }\end{array}$ & $\begin{array}{l}\text { PRECIO } \\
\text { USD/BI }\end{array}$ & $\begin{array}{r}\text { VALOR } \\
\text { Millones } \\
\text { USD }\end{array}$ & $\begin{array}{r}\text { VOLUMEN } \\
\text { Millones } \\
\text { Bls. }\end{array}$ & $\begin{array}{l}\text { PRECIO } \\
\text { USD/BI }\end{array}$ & $\begin{array}{r}\text { VALOR } \\
\text { Millones } \\
\text { USD }\end{array}$ \\
\hline I Trimestre & 34.98 & 43.95 & $1,537.26$ & 31.11 & 58.42 & $1,817.35$ \\
\hline II Trimestre & 34.67 & 42.13 & $1,460.56$ & 32.08 & 62.01 & $1,989.32$ \\
\hline III Trimestre & 34.02 & 43.53 & $1,480.88$ & 34.05 & 64.79 & $2,205.82$ \\
\hline IV Trimestre & 31.83 & 53.76 & $1,711.12$ & 32.46 & 56.72 & $1,840.92$ \\
\hline TOTAL & 135.49 & 45.68 & $6,189.82$ & 129.69 & 60.55 & $7,853.41$ \\
\hline
\end{tabular}

Fuente: Reporte del sector petrolero 2018 BCE

Es importante mencionar que dentro del universo empresarial del Ecuador, una buena proporción de empresas corresponde al RISE, En el cuadro xxx se puede notar que del total de empresa de empresas, equivale al $49 \%$ en 2017 y a $48 \%$ en 2018, es decir que casi alcanzan la mitad del total de empresas registradas según su forma institucional, por lo cual el monto recaudado por este rubro debería ser más significativo. 


\section{Cuadro 3}

Estructura de empresas según forma institucional, Año 2017-2016.

\begin{tabular}{||l|l|l|}
\hline \hline Forma institucional & 2017 & 2018 \\
\hline Total & 884.236 & 899.202 \\
\hline Régimen simplificado RISE & 437.258 & 429.202 \\
\hline Persona Natural no obligada a llevar contabilidad & 289.546 & 307.018 \\
\hline Sociedad con fines de lucro & 75.416 & 79.313 \\
\hline Perona natural obligada a llevar contabilidad & 49.695 & 49.158 \\
\hline Institución pública & 15.944 & 15.808 \\
\hline Sociedad sin fines de lucro & 11.985 & 12.735 \\
\hline Otros sectores institucionales & 4.392 & 5.968 \\
\hline
\end{tabular}

Fuente.: Directorio de empresas 2018 INEC

\section{Estructura de empresas según forma institucional}

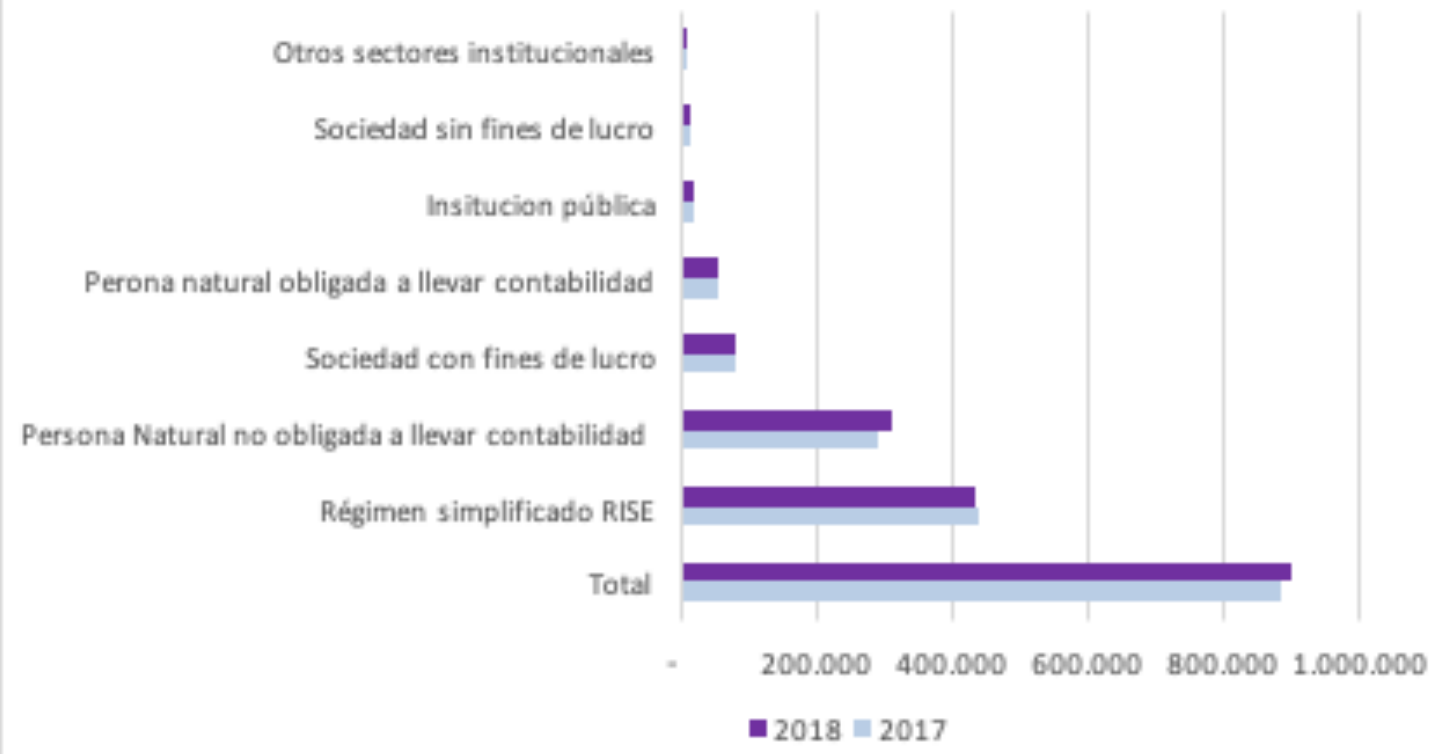

Figura 1. Estructura de empresas según forma institucional (2017-2018)

Fuente: Elaboración propia con datos del Directorio de empresas 2018 INEC 


\section{Discusión}

Se observa que la mayor proporción de impuestos son los correspondientes al Impuesto a la renta y al IVA, los cuales en conjunto alcanzan aproximadamente el $67 \%$ del total de la recaudación en los dos años estudiados

Puede notarse que aún existe una proporción de impuestos derivados de intereses de mora y multas tributarias, lo cual denota que falta aún por mejorar la cultura tributaria del país. Es de recalcar que los intereses por mora tributaria se incrementaron más del doble de un año a otro

Además, dada la gran parte de empresas bajo el régimen RISE, debería existir una mayor proporción de recaudación por este rubro.

El Ecuador a raíz de la volatilidad de los precios del petróleo, ha pasado a dar mayor importancia a la recaudación de los impuestos de sus conciudadanos y el mayor control tributario, se evidencia que al contrastar los ingresos fiscales por recaudación tributaria con los ingresos por exportaciones nacionales de crudo (petróleo) en el año 2017 ascienden a más de 13 mil millones de dólares los primeros y a solo un poco más de 6 mil millones los segundos; y en el año 2018 alcanzan más de 15 millones los primeros y a más de 7 mil millones los segundos. Es decir que los ingresos fiscales por $r$

\section{Conclusiones}

- Para la sostenibilidad fiscal del Ecuador se evidencia la relevancia de la recaudación tributaria puesto que el monto anual duplica los ingresos petroleros, los cuales además de ser menores, son muy volátiles.

- Los impuestos con mayor recaudación en el país son el impuesto a la Renta, y el IVA y sin embargo, hay otros impuestos que todavía no se los puede recaudar a cabalidad, es importante que el SRI busque mecanismos para mejorar esta recaudación o elimine definitivamente estos impuestos

- La proporción de recaudación por intereses de mora y por multas es mayor que la recaudada por RISE, aunque las empresas bajo este régimen conforman casi el 50 del total de empresa en el Ecuador,

- lo cual implica que se debe fomentar una buena cultura tributaria en función de que todos los individuos que conforman la sociedad tomen conciencia y cumplan con sus obligaciones constitucionales y las cumplan, eso ayuda al Estado a cumplir mejor con su rol de satisfacer necesidades públicas.

- Es obligación del Estado comunicar, mantener al día sobre los cambios legales y tributarios a toda su sociedad, para así lograr armonía en cuestiones tributarias.

- Es casi imposible detectar cuando se ha incumplido un pago de tributos de manera intencional, 
sin embargo, otra de las atribuciones del SRI, es la de sancionar en cualquiera de los casos, ya que el desconocimiento de la ley no exime de culpas y como todos los ciudadanos se los debe tratar en un ámbito de igualdad de igual manera se obtiene sanción.

- Los cambios drásticos que se han dado a nivel de control, reglamentación y sobre todo concienciación deben servir para que los Contribuyentes de todo el país comprendan la importancia de contribuir en el desarrollo de los planes de Gobierno. 


\section{REFERENCIAS BIBLIOGRÁFICAS}

Bernal, C. (2010). Metodología de la investigación. 3ed. PEARSON.

Código Tributario. Registro Oficial Suplemento 38 de 14-jun.-2005 Reformado 21-ago.-2018

Conferencia técnica Administración Tributaria Viable, Estado Viable. Centro Interamericano de Administraciones Tributarias - CIAT, (2005) Recuperado de https://www.ciat.org/Biblioteca/ConferenciasTecnicas/2005/Espanol/cartagena_2005_tema_3_1_charry_colombia.pdf

Cultura tributaria (6 de julio 2012(Entrada de blog) Recuperado de http://impuestos43.blogspot.com/2012/07/ que-es-cultura-tributaria.html

Directorio de empresas 2018 INEC Recuperado de https://www.ecuadorencifras.gob.ec/directoriodeempresas/

Maldonado P. y De la Torre C. (2016) Quienes son Personas Naturales no obligadas a llevar contabilidad. Contabilidad y Auditoría Recuperado de http://www.contadoresenquito.com/noticias-contadores-economia-trabajos-auditorias-contable-empresas-quito-manabi-santo-domingo-ecuador.php?tablajb=noticias\&p=76\&$\mathrm{t}=$ Quienes-son-Personas-Naturales-no-obligadas-a-Ilevar-contabilidad\&

Reporte del sector petrolero 2018 BCE Recuperado de https://contenido.bce.fin.ec/documentos/Estadisticas/ Hidrocarburos/ASP201812.pdf

Romero, A (s/f) Instituciones públicas (2018). Diccionario ley derecho. Recuperado de https://diccionario.leyderecho.org/instituciones-publicas/

Servicio de Rentas Internas SRI (s.f.). Información General Obtenido de https://www.sri.gob.ec/web/guest/informacion-general

Servicio de Rentas Internas SRI (s/f) Estadísticas Generales de Recaudación https://www.sri.gob.ec/web/guest/ estadisticas-generales-de-recaudacion

Servicio de Rentas Internas SRI (s/f) RISE. Recuperado de https://www.sri.gob.ec/web/guest/regimen-impositivo-simplificado-rise

Thompson L. (2007) Tipos de organizaciones. Recuperado de https://www.promonegocios.net/empresa/tipos-organizaciones.html 